\title{
A method of power system simulation model reduction for transmission grid frequency response analysis
}

\author{
Valery Solodovnikov ${ }^{1, *}$, Vladimir Tulsky ${ }^{1}$, and Roman Shamonov ${ }^{2}$ \\ ${ }^{1}$ NRU "Moscow Power Engineering Institute", Moscow, Russian Federation \\ ${ }^{2}$ PJSC "FGC UES", Moscow, Russian Federation
}

\begin{abstract}
Application of the frequency scan method for the determination of resonant conditions in a transmission power grid requires great effort since the harmonic power system simulation model needs to be developed. This process is rather complicated since the original model used for fundamental frequency load-flow analysis is built with respect to certain assumptions and, thus, intolerable errors are introduced when frequency-domain properties of the power system are investigated. To strike a balance between inputs needed for the development of such model (time, data amounts) and accuracy of the results, it is proposed to employ a method which makes it possible to represent dead-end, double-ended and tapped 110-220 kV substations as a single frequency-dependent equivalent so that the harmonic power system model is reduced. Such an element essentially is a series $R-L$ or $R-L-C$ shunt, parameters of which vary with frequency. The algorithm for the evaluation of its parameters is proposed and the test case for a real $110 \mathrm{kV}$ grid area is discussed. Results of the method application show that it can be used in practice.
\end{abstract}

\section{Introduction}

Frequency scan (FS) is regarded as one of the most widely used methods for the investigation of harmonic voltage level variation trends, which consists in the estimation of the frequency response of power system positive (negative) sequence impedance as seen from the nodes of an electrical grid and subsequent identification of resonant frequencies [1-4]. As outlined in [1, 4, 5], development and verification of a power system simulation model is by far the most time- and laborconsuming stage of the investigation when $220 \mathrm{kV}$ and above transmission grid is of concern. It is due to the fact that $110-220 \mathrm{kV}$ grid areas, which do not have a significant impact on a power system fundamental frequency load-flow, are usually modelled as equivalent power take-offs, while valid frequency response can only be obtained if such areas are fully incorporated into a power system model $[1,4]$. For this reason, considerable enhancement of the original fundamental frequency power system model is required in order to perform FS, resulting in drastic increase in its size defined by the number of nodes and branches. Therefore, maintenance and practical use of the derived harmonic model becomes rather difficult, which makes the assurance of acceptable $K_{U}$ (voltage total harmonic distortion) and $K_{U(n)} \quad\left(n^{\text {th }}\right.$ harmonic voltage factor) levels in a transmission grid a rather complicated task.

Since over-limit voltage harmonic distortion is a topical issue for Russian grid utilities [6], a method of power system simulation model reduction is proposed in this article. The method makes it possible to represent dead-end, double-ended and tapped 110-220 kV substations as frequency-dependent equivalents (FDEs) when FS is performed at the $110 \mathrm{kV}$ and above buses of the transmission grid substations.

\section{Frequency-dependent equivalents of 110-220 kV substations}

\subsection{Use of frequency-dependent equivalents for frequency response analysis: motivation}

Frequency response of the driving-point impedance as seen from the nodes of a $220 \mathrm{kV}$ and above transmission grid strongly depends on the actual topology of adjacent distribution grids (primarily 110 and $150 \mathrm{kV}$ ) as well as on the parameters of their elements, which makes it necessary to incorporate them into harmonic power system model $[1,4]$. On the other hand, power system models used for the load-flow analysis at the fundamental frequency (i.e. for the purposes of transmission grid operational control) are usually developed with the following simplifications taken into account:

- 110 (150) kV dead-end grid fragments can be modelled as equivalent power take-offs provided that bus voltage levels and line ampacity do not exceed permissible values during both normal operation and contingencies;

- 110-220 kV substations can be represented as equivalent loads referred to $\mathrm{HV}$ side in case no reactive power compensators are installed or their output is relatively small;

* Corresponding author: valerysol@yandex.ru 
- 110-220 kV substations can be represented as equivalent loads referred to HV side unless generation units are connected to MV or LV buses either directly or via dead-end grid fragments.

Therefore, harmonic power system model can be built on the basis of existing fundamental frequency model, but the latter needs to be expanded significantly so that the valid FS results could be obtained. It is thus proposed to represent dead-end, double-ended and tapped 110-220 kV step-down substations as FDEs in order to minimize harmonic model size increase.

\subsection{Composite harmonic model of a $110-220 \mathrm{kV}$ substation}

Composite harmonic model of 110-220 kV step-down substations include the following elements:

- power transformer;

- load;

- reactive power compensation units and filters.

Power transformers in the frequency range from $2^{\text {nd }}$ to $40^{\text {th }}$ harmonic are represented as an equivalent circuit consisting of resistive and inductive elements connected in series or series-parallel manner; excitation circuit is usually neglected [1-4].

In accordance with [1, 3-5], actual load composition must be taken into account when performing harmonic assessments in power systems. However, gathering such information for composite loads fed from 110-220 kV substations is a challenging task since standard-form connection contracts and technical specifications stated by [8] impose no customer obligations regarding provision of contracted capacity disaggregated by receiver type during connection procedure. For this reason, aggregate load models are used for FS.

Such models proposed in [1-5] are essentially passive one-ports, parameters of which can be evaluated given the fundamental frequency voltage at the connection point, active power demand and two consolidated values, namely electronic and motor fractions of the total active power demand. Besides, reactive power demand can be used for evaluation of parameters in case motor fraction is less than $10 \%$. It is also noteworthy that load model proposed in [4] also accounts for the total capacitance of outgoing 6-35 kV feeders.

Driving-point impedance frequency response is also heavily influenced by reactive power compensation units and filters in service. Such devices are represented in a harmonic power system model as a shunt inductance or capacitance $[1-4,7]$.

Hence, a 110-220 kV step-down substation feeding composite load can be modelled as a passive one-port in the frequency range of interest. The impedance of such a one-port is determined by its topology and parameters of the constituent elements and, in return, can be associated with a series $R-L$ shunt in case of a substation feeding lagging load. If reactive power compensation units installed at the substation are in service and/or distribution grid feeders' capacitance is taken into account, the model can be extended to $R-L-C$ shunt (see
Fig. 1). Generally, parameters of the equivalent shunt are frequency-dependent.

Representation of $110-220 \mathrm{kV}$ substations in a harmonic power system model as FDEs of such a specific topology is required in case impedance with reversal imaginary part cannot be modelled as a single element by means of a software tool used for FS.

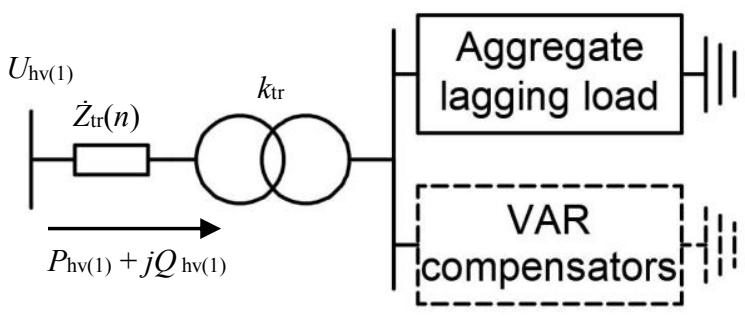

a)

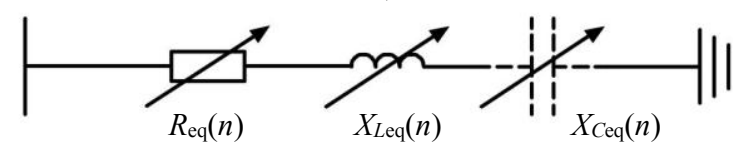

b)

Fig. 1. Frequency-dependent equivalent of a $110-220 \mathrm{kV}$ single-transformer step-down substation: a) - original two-port; b) - equivalent shunt.

\subsection{Description of the proposed method}

According to recommendations given in [4], frequency response assessment of the driving-point impedance as seen from transmission grid nodes is usually performed under the assumption that a power system is perfectly balanced in terms of both circuit and state parameters, which makes it possible to employ single-phase approach. In that respect, since the fundamental frequency steady-state of the original substation one-port is determined by the voltage and apparent power flow at the transformer HV side, parameters of an FDE can be calculated by means of the method described below (see Fig. 2 for the algorithm flowchart).

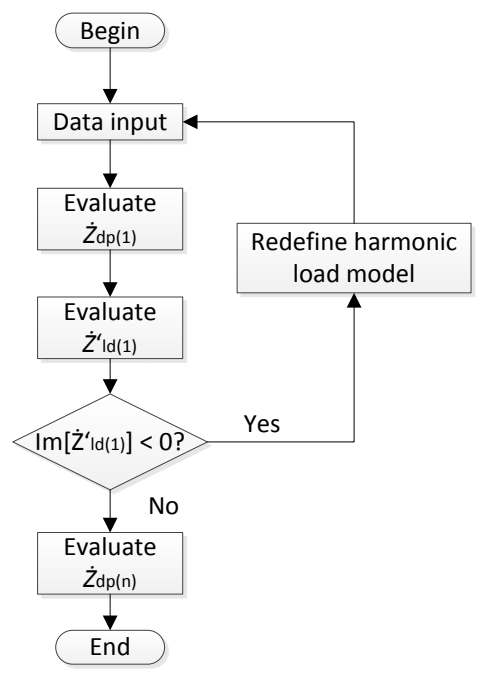

Fig. 2. Flowchart of the algorithm for the evaluation of parameters of a 110-220 kV substation frequency-dependent equivalent. 
The input data are as follows:

- Fundamental frequency load-flow parameters:

- phase-to-phase voltage at the HV bus $U_{\text {hv(1) }}$;

- three-phase apparent power demand referred to the $\mathrm{HV}$ side $P_{\mathrm{hv}(1)}+\mathrm{j} Q_{\mathrm{hv}(1)}$.

- Transformer parameters:

- OLTC tap position;

- fundamental frequency impedance at the given tap position $\dot{Z}_{\mathrm{tr}(1)}$;

- turns ratio at the given tap position $k_{\mathrm{tr}}$.

- Load parameters:

- fraction of the electronic load $K_{\mathrm{E}}$;

- fraction of the motor load $K_{\mathrm{MT}}$ (if considered);

- total capacitance of outgoing $6-35 \mathrm{kV}$ feeders (if considered)

- Reactive power compensator parameters:

- total reactance of reactive power compensators in service (if any).

Bus voltage and apparent power at the fundamental frequency are obtained on the basis of the preliminary load-flow analysis. Transformer parameters are defined given its nameplate data. It is also recommended to use actual data on load composition; however, average $K_{\mathrm{E}}$ and $K_{\mathrm{M}}$ values presented in [7,9] can be used if such information is unavailable.

Additional information is needed in case threewinding and split-winding transformers are installed at the substation:

- HV, MV and LV transformer branch impedance at the given OLTC and DETC tap positions;

- active and reactive power demand ratio on the MV and LV sides respectively (can be assumed with respect to check measurements).

For simplicity, the algorithm is further described for a substation with a single two-winding transformer as an example.

Step 1. Equivalent driving-point impedance of the substation one-port is calculated by the formula (1):

$$
\mathbb{Z}_{\mathrm{dp}(1)}=\left(P_{\mathrm{hv}(1)}+j Q_{\mathrm{hv}(1)}\right) \cdot \frac{U_{\mathrm{hv}(1)}^{2}}{P_{\mathrm{hv}(1)}^{2}+Q_{\mathrm{hv}(1)}^{2}}
$$

Step 2. Fundamental frequency load impedance referred to the HV side is evaluated. If no reactive power compensation units are in service, the impedance can be obtained from equation (2):

$$
\mathbb{Z}_{\mathrm{ld}(1)}^{\&}=\mathbb{Z}_{\mathrm{dp}(1)}^{\&}-\mathbb{Z}_{\mathrm{tr}(1)}^{\&}
$$

In presence of reactive power compensators load impedance can be found by the formula (3), where $X_{\mathrm{vcu} \Sigma}$ is the total reactance of compensators referred to the $\mathrm{HV}$ side:

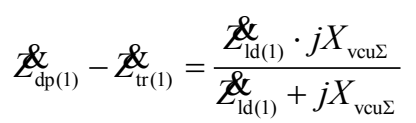

Step 3. If the reactive power demand is used for the evaluation of the load model parameters, the value of the imaginary part of $\dot{Z}^{\prime} \operatorname{ld}(1)$ is then analyzed. A negative value indicates that either a more sophisticated load model is required to determine FDE parameters or the adjacent area of the $35 \mathrm{kV}$ and below distribution grid needs to be represented in the harmonic power system model.

It is essential to test this condition since models proposed in $[1,5]$, parameters of which are derived from the reactive power, are valid in case of the inductive load. In real operation, a load might be capacitive due to the following reasons:

- motive load operating conditions (i.e. synchronous motors with a leading power factor);

- presence of the capacitor banks in the downstream distribution grid;

- light-load conditions and/or significant capacitance of outgoing 6-35 kV feeders.

Step 4. FDE parameters are evaluated in the frequency range of interest. Design formulae are determined by load and power transformer models as well as presence of reactive power compensators. For instance, if models presented in Fig. 3 and proposed in [1] are adopted and no compensators are installed, equivalent parameters at the $n^{\text {th }}$ harmonic frequency can be found from equations (4) and (5):

$$
\begin{aligned}
& R_{\mathrm{eq}}(n)=\frac{1}{1-K_{\mathrm{E}}-K_{\mathrm{MT}}} \cdot \frac{\left|\mathcal{E}_{\mathrm{ld}(1)}^{\&}\right|^{2}}{\operatorname{Re}\left[\mathbb{Z}_{\mathrm{ld}(1)}^{\&}\right]} \cdot \frac{n^{2} K_{1}^{2}}{1+n^{2} K_{1}^{2}}+R_{\mathrm{tr}(n)} \\
& X_{L \text { eq }}(n)=\frac{1}{1-K_{\mathrm{E}}-K_{\mathrm{MT}}} \cdot \frac{\left|\mathbb{E}_{\mathrm{ld}(1)}^{\&}\right|^{2}}{\operatorname{Re}\left[\mathbb{Z}_{\mathrm{ld}(1)}^{\&}\right]} \cdot \frac{n K_{1}^{2}}{1+n^{2} K_{2}^{2}}+X_{\mathrm{tr}(n)}
\end{aligned}
$$

Capacitive reactance $X_{C \text { eq }}$ is set to zero since chosen load model does not account for the impact of outgoing feeders. Transformer parameters at the $n^{\text {th }}$ harmonic frequency and $K_{1}$ factor can be calculated as follows:

$$
\begin{gathered}
R_{\operatorname{tr}(n)}=R_{\operatorname{tr}(1)}+R_{\operatorname{tr}(1)} \cdot \frac{10 n^{2}}{100+\left(\frac{n R_{\operatorname{tr}(1)}}{X_{\operatorname{tr}(1)}}\right)^{2}} \\
X_{\operatorname{tr}(n)}=\frac{100 \cdot X_{\operatorname{tr}(1)} \cdot n}{100+\left(\frac{n R_{\mathrm{tr}(1)}}{X_{\operatorname{tr}(1)}}\right)^{2}} \\
K_{1}=\frac{X_{\mathrm{M}} \cdot\left(1-K_{\mathrm{E}}-K_{\mathrm{MT}}\right)}{K_{\mathrm{MT}} \cdot K_{m}}
\end{gathered}
$$

Names of the variables in the last equation are assumed in accordance with [1].

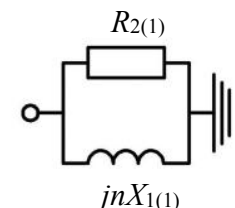

Fig. 3. Examples of load and two-winding power transformer harmonic models: IEEE 'Induction motors' load model (left), Electra-167 transformer model (right).

It should be noted that each one-port consisting of a power transformer as well as loads and reactive power compensation units connected to it are replaced with its own FDE. Therefore, it is reasonable to represent multiple-transformer substation in the harmonic power system model as a single equivalent. Its parameters are estimated in the frequency range of interest as the total impedance of equivalent shunts connected in parallel. 
As a result, dead-end, double-ended and tapped 110$220 \mathrm{kV}$ step-down substations can be represented as FDEs by means of the proposed method. The advantages of the method are as follows:

- FDE parameters are determined directly from the load-flow parameters on the HV side, which makes it possible to build harmonic power system model with minimum adjustment of the original fundamental frequency model and, therefore, to simplify its development, practical use and maintenance;

- representing FDEs as series $R-L(R-L-C)$ shunts facilitates application of the method since such elements are basic for harmonic analysis software tools.

\section{Simplified representation of the 110- $220 \mathrm{kV}$ substations}

Under certain conditions, simplified representation of $110-220 \mathrm{kV}$ substations in the harmonic power system model is possible due to the frequency-domain properties of load and transformer models.

According to [5], an aggregate load can be modelled as a series $R-L$ shunt if the motor fraction is small (less than $10 \%)$. If reactive power compensation units are not installed at the substation, a transformer can be excluded from the original 'transformer + load' one-port given the specific combination of load-flow parameters at the fundamental frequency. Therefore, the original one-port can be replaced by the series $R-L$ shunt; its parameters are estimated given the HV bus voltage and apparent power on the HV side at the fundamental frequency.

It must be emphasized that the discussed conversion can be regarded as equivalent in terms of electric circuit theory only at the fundamental frequency. Thus, conversion acceptance criteria were defined. The criteria (9) state that the impedance modulus relative difference for the original and converted one-ports (amplitudefrequency response, AFR) as well as impedance angle difference (phase-frequency response, PFR) does not exceed $5 \%$ in the frequency range of interest:

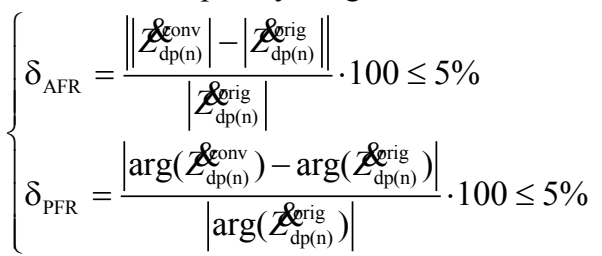

It is then possible to assess permissibility of the simplified representation of $110-220 \mathrm{kV}$ substations under given load-flow conditions at the fundamental frequency. Based on the above-stated criteria, a set of load-flow parameters can be divided into regions where the simplification is and is not justifiable.

As an example, such regions are presented in the Fig. 4 for the substations with a single $110-220 \mathrm{kV}$ splitwinding transformer installed. Frequency-dependence of the transformer impedance is taken in accordance with IEEE-399 [10], loads connected to secondary windings LV1 and LV2 are assumed to be linear.

Simplified substation representation is allowed if the transformer operating point lies below the border drawn in the 'p.u. transformer load - maximum $\operatorname{tg} \varphi$ of LV1 and LV2 loads' plane. Boundaries presented in Fig. 4 are valid under the following conditions:

- transformers are manufactured in accordance with regulations $[11,12]$;

- fundamental frequency voltage at the $\mathrm{HV}$ bus is within the range of $0.8 U_{\text {nom }}$ to maximum operating voltage based on the requirements [13].

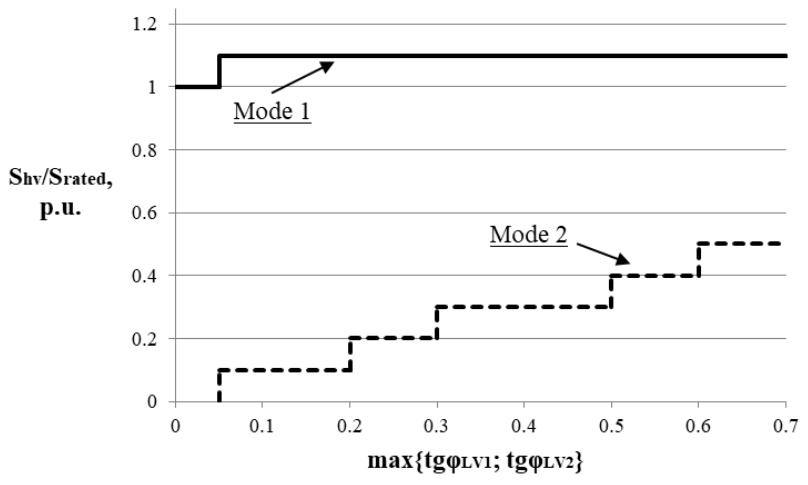

Fig. 4. Simplified representation regions for the substations with a single $110-220 \mathrm{kV}$ split-winding transformer installed.

Since AFR and PFR of the original one-port impedance are heavily influenced by the ratio between loads connected to transformer secondary windings, boundaries are defined for two operating modes:

- $\quad$ Mode 1: even load distribution $\left(S_{\mathrm{LV} 1}=S_{\mathrm{LV} 2} ; \operatorname{tg} \varphi_{\mathrm{LV} 1}=\right.$ $=\operatorname{tg} \varphi_{\mathrm{LV} 2} ;$ solid line);

- Mode 2: uneven load distribution $\left(S_{\max } / S_{\min } \leq 2\right.$; $\operatorname{tg} \varphi_{\text {Smax }} / \operatorname{tg} \varphi_{\text {Smin }} \leq 1,5$; dashed line).

It can be seen from Fig. 4 that load ratio has a significant impact on the permissibility of the simplified representation of substations in a harmonic power system model. If loads are distributed evenly between transformer secondary windings, a simplification can be implemented over the wide range of transformer load (from no-load operation to $1.0-1.1 S_{\text {rated }}$ ) and $\operatorname{tg} \varphi$ (from 0 to 0.7$)$ values. On the contrary, the allowable region shrinks if loads are unequal: a conversion can be performed in case transformer load does not exceed 0.3 $0.5 S_{\text {rated }}$ (the former value corresponds to the $\operatorname{tg} \varphi_{S \max }$ between 0.05 and 0.4 , the latter - between 0.6 and 0.7 ).

It should be noted that if the impact of transformer outages on the transmission grid frequency response is of interest, the assessment of simplified representation permissibility for $110-220 \mathrm{kV}$ substations with 2 or more transformers installed should be performed in case of an outage due to increase in power flow through the transformers left in operation.

\section{Test of the method: a real-life $110 \mathrm{kV}$ grid area case study}

The proposed method was tested for an area of a $110 \mathrm{kV}$ grid; its simplified one-line diagram is presented in Fig. 5. Power is supplied from the $220 \mathrm{kV}$ substation $\mathrm{K}$, which is electrically close to $500 \mathrm{kV}$ transmission grid 
facilities. The area of interest consists of dead-end fragments connected to substation $\mathrm{K} 110 \mathrm{kV}$ buses and interconnector between substations $\mathrm{K}$ and $\mathrm{A}$, which feeds tapped and double-ended $110 \mathrm{kV}$ substations. The interconnector is normally open-circuited (substation $15110 \mathrm{kV}$ bus coupler and line circuit breakers at the substation $\mathrm{C}$ are open). Total length of the interconnector is $302 \mathrm{~km}$; the section between substations $\mathrm{K}$ and 15 is $117 \mathrm{~km}$ long.

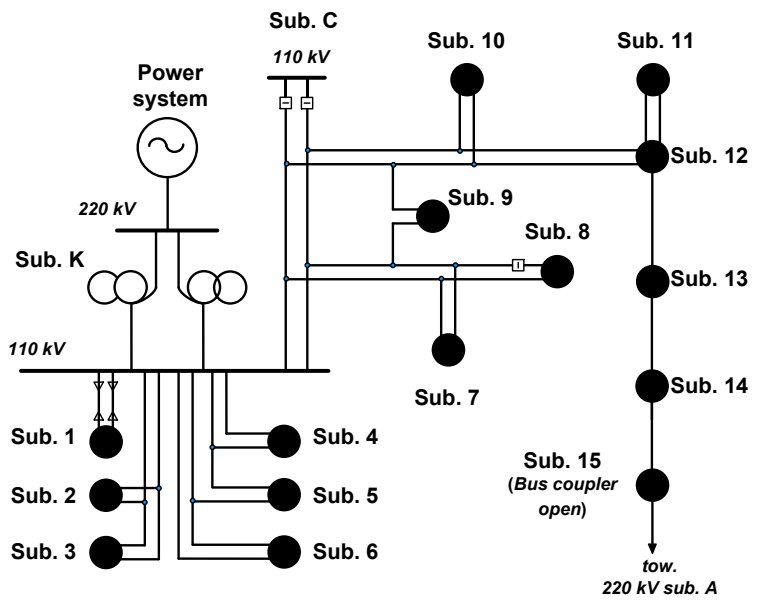

Fig. 5. Simplified one-line diagram of the $110 \mathrm{kV}$ grid analysis area for the test of the proposed method.

FS was performed to obtain AFR and PFR of the driving-point impedance as seen from the $110 \mathrm{kV}$ bus of substation $\mathrm{K}$. This grid point is of interest since it defines a boundary between areas of responsibility of regional distribution and transmission grid operators.

Driving-point impedance AFR and PFR curves as at 10:00 06/20/2018 (Moscow time zone, summer check measurement day) are presented in Fig. 6. Total area load demand amounted to $67.8+j 27.6$ MVA. Curves are plotted for three types of a harmonic power system simulation model:

- detailed model (all the $110 \mathrm{kV}$ transformers are represented) - 106 nodes, 141 branches;

- FDE representation - 50 nodes, 64 branches;

- simplified representation of $110 \mathrm{kV}$ substations 7-9.

It should be noted that curves are plotted only for the first and the last case since FDE representation is strictly equivalent in the frequency range of interest. Therefore, AFR and PFR curves for the first and second case are essentially the same (relative deviation does not exceed $0.05 \%$ and is caused by rounding errors).

At the same time, simplified representation of substations 7-9 causes moderate decrease in impedance modulus close to the first resonant frequency (less than $2 \%$, circled in the Fig. 6) and introduces slight upwards shift $(5 \mathrm{~Hz})$ of such frequency.

It can be seen that application of the proposed method makes it possible to cut down number of nodes and branches more than by half (from 106 down to 50 and from 141 to 64 respectively) with the same computational accuracy. Simplified representation introduces light errors; however, they are offset by input data reduction since gathering and processing of the 110-
$220 \mathrm{kV}$ transformer data is not required in this case, which, eventually, makes it possible to speed up the transmission grid frequency response analysis.

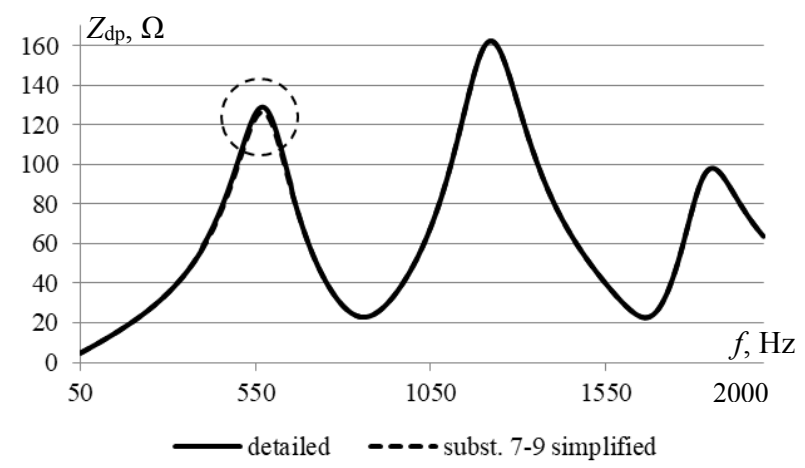

a)

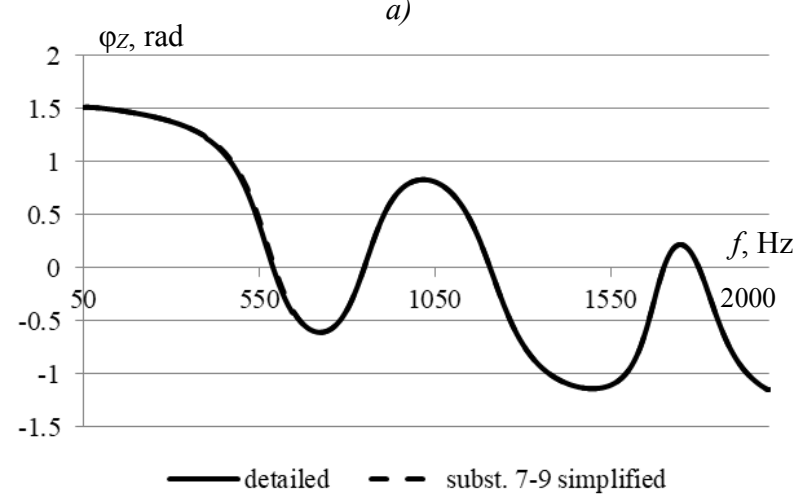

b)

Fig. 6. Positive (negative) sequence driving-point impedance frequency response as seen from the $110 \mathrm{kV}$ buses of the 220 $\mathrm{kV}$ substation K: a) - AFR curve; b) - PFR curve.

\section{Conclusions}

Based on the foregoing, the following conclusions can be made:

1. Development and verification of a power system simulation model is the most time- and labor-consuming stage of the harmonic voltage trend investigation when the transmission grid is of concern since 110-220 kV grid areas, which do not have a significant impact on a power system fundamental frequency load-flow, are usually modelled as equivalent power take-offs, while valid frequency response can only be obtained if such areas are fully incorporated into a power system model.

2. Dead-end, double-ended and tapped 110-220 kV step-down substations can be modelled as frequencydependent equivalents (FDE) unless generation units are connected to MV or LV substation buses either directly or via dead-end grid fragments. The FDE is a series $R-L$ or $R-L-C$ shunts with frequency-dependent parameters. Such a specific representation is required in case impedance with reversal imaginary part cannot be modelled as a single element by means of a software tool used for FS.

3. A method of harmonic power system model reduction is proposed, which makes it possible to employ FDEs for the purposes of FS instead of the full 
substation representation. FDE parameters are estimated on the basis of the fundamental frequency load-flow parameters at the beginning of a transformer branch. Therefore, the size of a harmonic power system model can be reduced, which simplifies its development, practical use and maintenance.

4. It is demonstrated that if aggregate load fed from the substation is modelled as a series $R-L$ shunt, simplified representation of such a substation is allowed under certain fundamental frequency conditions. In this case, substation as a whole can be modelled as a series $R-L$ shunt; its parameters are evaluated given the HV bus voltage and apparent power flow; transformer impedance is neglected. Permissibility analysis is performed on the basis of plots which reflect the possible transformer operating conditions: if the operating point lies below the border defined according to permissibility criteria, a substation can be represented in a simplistic manner.

5. The proposed method of harmonic power system model reduction was tested for a real-life $110 \mathrm{kV}$ grid area. It is shown that the number of nodes and branches can be reduced more than by half if FDEs are employed. Simplified representation introduces moderate errors, but, at the same time, data amount needed for the performance of FS is reduced, which speeds up the analysis.

\section{References}

1. M. Val Escudero, Z. Emin, G. Lietz, C.F. Jensen et al., CIGRE TB № 766 'Network modelling for harmonic studies' (CIGRE, 2019)

2. J. Arrillaga, N.R. Watson, Power system harmonics. Second Edition (John Wiley \& Sons Ltd., Chichester, 2003)

3. J.C. Das, Power system harmonics and passive filter designs (IEEE Press, Picastaway, 2015)

4. S.S. Smirnov, Higher harmonics in high-voltage grids (Nauka, Novosibirsk, 2010)

5. R. Burch et al., IEEE Trans. Power Deliv. 18, 626 (2003)

6. L.I. Kovernikova, V.V. Sudnova, R.G. Shamonov et al., Power quality: state-of-the-art, problems and solution proposals (Nauka, Novosibirsk, 2017)

7. I.I. Kartashev, V.N. Tulsky, R.G. Shamonov et al., Power quality management (MPEI publishing house, Moscow, 2017)

8. Decree of the Government of the Russian Federation No. 861 of December 27, 2004 "On approval or Regulation of non-discriminatory access to electricity transmission grid..."

9. RD 153-34.0-20.527-98 "Guidelines for calculation of short circuit currents and selection of electrical equipment"

10. IEEE Std. 399-1997 "IEEE recommended practice for industrial and commercial power systems analysis"

11. GOST 12965-85 "General-purpose 110 and $150 \mathrm{kV}$ power oil transformers. Specifications
12. GOST 17544-85 “General-purpose 220, 330, 500 and $750 \mathrm{kV}$ power oil transformers. Specifications"

13. Decree of the Government of the Russian Federation No. 937 of June 13, 2018 "On the approval of Guidelines for electrical power system technical functioning" 